Pacific Journal of Mathematic 


\title{
MONODROMY AND INVARIANTS OF ELLIPTIC SURFACES
}

\author{
Peter F. Stiller
}

The purpose of this research is to analyze and compute the monodromy representation of the Gauss Manin connection associated with an elliptic surface and to relate properties of the monodromy to geometric properties of the surface. The results utilize the general theory of elliptic surfaces due to Kodaira.

Let $E$ be an elliptic surface having a global section over its base curve $X$. We assume throughout that the functional invariant $\mathscr{J}$ is nonconstant and that $E$ has no exceptional curves of the first kind in the fibres. We denote by $G$ the homological invariant of $E / X$. On a Zariski open subset $X_{0} \subset X, G$ can be viewed as either a locally constant $\boldsymbol{Z} \oplus \boldsymbol{Z}$ sheaf or as a representation $\pi_{1}\left(X_{0}\right) \rightarrow \mathrm{SL}_{2}(\boldsymbol{Z})$. This representation corresponds to an algebraic vector bundle of rank two on $X$ together with an integrable algebraic connection having regular singular points (Deligne [1], Griffiths [2]), which is known as the Gauss-Manin connection (Katz and Oda [4]). It can be expressed as a second order algebraic differential equation on $X$ having regular singular points. The explicit form of this equation that we shall make use of appears in Stiller [12].

We begin with a brief section of preliminaries, recalling some previous results which relate the geometry of the elliptic surfaces over $X$ to properties of the corresponding differential equations ( $K$ equations, see Stiller [12]).

The first section describes a period mapping from the base curve $X$ to the modular curve $M_{\Gamma}$ where $\Gamma \subset \mathrm{SL}_{2}(\boldsymbol{Z})$ is the global monodromy group of both $E / X$ and the differential equation. Also we give a number of conditions under which $\Gamma=\mathrm{SL}_{2}(\boldsymbol{Z})$ (see also $\S 3$ ). When $\Gamma=\mathrm{SL}_{2}(\boldsymbol{Z})$ the group of $K(X)$-rational division points on the generic fibre (which is an elliptic curve over $K(X)$ the function field of the base curve $X$ ) is zero.

In section two we examine a number of invariants of $E / X$ such as the Picard number, the valence of the functional invariant $\mathscr{J}$, the index of the monodromy group $\Gamma$ in $\mathrm{SL}_{2}(\boldsymbol{Z})$, and other numerical invariants to determine their behavior when we pass to a generically isogeneous surface over $X$. The main results are that all of these invariants remain unchanged under generic isogeny! We will utilize the fact that in this case the differential equation does not change (Stiller [12]). 
We finish by giving a method for determining the monodromy representation and computing several examples which illustrate the results.

O. Preliminaries. Let $X$ be a complete smooth connected curve over $C$ with function field denoted by $K(X)$. After fixing a parameter $x \in K(X)$, consider an algebraic differential equation on $X$

$$
\Lambda f=\frac{d^{2} f}{d x^{2}}+P \frac{d f}{d x}+Q f=0
$$

with $P$ and $Q$ in $K(X)$ and $f$ an unknown function.

Definition 0.1. $\Lambda f=0$ is called a $K$-equation if it possesses two solutions, $\omega_{1}$ and $\omega_{2}$, which are holomorphic nonvanishing multivalued functions on some Zariski open subset $X_{0}$ of $X$, satisfying:

(i) $\omega_{1}$ and $\omega_{2}$ form a basis of solutions,

(ii) for every closed path $\gamma \in \pi_{1}\left(X_{0}\right)$ the analytic continuation of $\left(\begin{array}{c}\omega_{1} \\ \omega_{2}\end{array}\right)$ around $\gamma$ is $M_{r}\left(\begin{array}{l}\omega_{1} \\ \omega_{2}\end{array}\right)$ with $M_{\gamma} \in \mathrm{SL}_{2}(\boldsymbol{Z})$ (the monodromy representation),

(iii) $\operatorname{Im}\left(\omega_{1} / \omega_{2}\right)>0$ on $X_{0}$ (positivity).

Such a pair of solutions is called a $K$-basis. In addition, since the monodromy is in $\mathrm{SL}_{2}(\boldsymbol{Z})$, the Wronskian $W=\exp \left(-\int P d x\right)$ is singlevalued. We assume as part of our definition:

(iv) $W \in K(X)$.

Let $A f=0$ be a $K$-equation with $K$-basis $\omega_{1}$ and $\omega_{2}$. Consider the function $\mathscr{f}=J \circ \omega_{1} / \omega_{2}$,

$$
X_{0} \stackrel{\omega_{1} / \omega_{2}}{\longrightarrow} \mathfrak{K} \stackrel{J}{\longrightarrow} C
$$

where $J$ is the elliptic modular function on the upper half plane $\mathfrak{S}$. This $\mathscr{J}$ is a single-valued holomorphic function on $X_{0} \subset X$.

Proposition 0.2. $\mathscr{J} \in K(X)$.

We now determine all $K$-equations. Fix a $K$-equation $\Lambda f=0$ on $X$ with $K$-basis $\omega_{1}, \omega_{2}$ such that $\mathscr{J}=J\left(\omega_{1} / \omega_{2}\right)$. Say

$$
\Lambda f=\frac{d^{2} f}{d x^{2}}+P \frac{d f}{d x}+Q f=0 .
$$

THEOREM 0.3. There exists an algebraic function $\lambda$ on $X$ with $\lambda^{2} \in K(X)$ such that 


$$
\begin{aligned}
P= & \frac{\left(\frac{d \mathscr{J}}{d x}\right)^{2}-\mathscr{J} \frac{d^{2} \mathscr{J}}{d x^{2}}}{\mathscr{J} \frac{d \mathscr{J}}{d x}}-\frac{d}{d x} \log \lambda^{2} \\
Q= & \frac{\left(\frac{d \mathscr{J}}{d x}\right)^{2}\left(\frac{31}{144} \mathscr{J}-\frac{1}{36}\right)}{\mathscr{J}^{2}(\mathscr{J}-1)^{2}}-\left(\frac{\left(\frac{d \mathscr{J}}{d x}\right)^{2}-\mathscr{J} \frac{d^{2} \mathscr{J}}{d x^{2}}}{\mathscr{J} \frac{d \mathscr{J}}{d x}}\right) \frac{d}{d x} \log \lambda \\
& +\frac{-\frac{d^{2} \lambda}{d x^{2}}}{\lambda}+2\left(\frac{\frac{d \lambda}{d x}}{\lambda}\right)^{2} .
\end{aligned}
$$

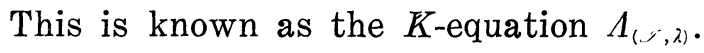

It is shown in Stiller [12] that $K$-equations are precisely those differential equations which arise naturally as the Gauss-Manin connexions associated to elliptic surfaces.

One can directly compute the local behavior of the solutions at the singularities of the differential equation (Ince [3], Picard [7]). The local monodromy matrix corresponds with the marix associated to the particular type of singular fibre of the elliptic surface; see Kodaira's list in Kodaira [5]. The reader unfamiliar with the relation between representations and differential equations, and the local properties of differential equations with regular singularities can consult Poincaré [8], Deligne [1], or Griffiths [2]. The only terminology that we employ which is not standard is that we refer to a singular point as cosingular if the solutions are single-valued meromorphic functions in a neighborhood of the point.

Given a $K$-equation $\Lambda$ on $X$ with $K$-basis $\omega_{1}, \omega_{2}$ one can construct a basic elliptic surface $E$ over $X$ with functional invariant $\mathscr{J}=$ $J\left(\omega_{1} / \omega_{2}\right)$ and homological invariant corresponding to the monodromy representation of $\Lambda$ using the basis $\omega_{1}, \omega_{2}$. There is no unique $K$ equation associated to a given elliptic surface $E / X$ and conversely a given $K$-equation may produce several surfaces for different choices of $K$-bases. However any other surface $E^{\prime} / X$ produced from the same $K$-equation $A$ via a different $K$-basis will be generically isogeneous to $E$, i.e., there will exist a rational map $\phi: E \rightarrow E^{\prime}$ over $X$ which over a Zariski open set $X_{0} \subset X$ will be a fibre by fibre isogeny. The converse also holds:

THEOREM 0.4. (Stiller [12].) Let E, E' be basic elliptic surfaces over $X$ which are generically isogeneous, then there is one K-equation $\Lambda$ with two $K$-bases $\omega_{1}, \omega_{2}$ and $\omega_{1}^{\prime}$, $\omega_{2}^{\prime}$ such that $E$ can be constructed from $\Lambda, \omega_{1}, \omega_{2}$, and $E^{\prime}$ can be constructed from $\Lambda, \omega_{1}^{\prime}, \omega_{2}^{\prime}$. Moreover 
since both $\omega_{1}, \omega_{2}$ and $\omega_{1}^{\prime}, \omega_{2}^{\prime}$ form bases of solutions for $\Lambda$ they are related by a constant matrix which is forced to be in $\mathrm{GL}_{2}^{+}(\boldsymbol{Q})$ as both are K-bases.

Thus if $E, E^{\prime}$ are generically isogeneous we have

$$
M \Gamma M^{-1}=\Gamma^{\prime}
$$

where $M \in \mathrm{GL}_{2}^{+}(\boldsymbol{Q})$ and $\Gamma, \Gamma^{\prime} \in \mathrm{SL}_{2}(\boldsymbol{Z})$ are the global monodromy groups of $E, E^{\prime}$ respectively. Of course if $M \in \mathrm{SL}_{2}(Z)$ or scalar then $E \cong E^{\prime}$.

In this way information about the entire isogeny class (of the generic fibre) is fixed in one differential equation. Any invariants which depend only on the differential equation are then the same for members of a given isogeny class. It is this idea that we shall pursue.

1. The monodromy. Let $X$ be a complete smooth curve over the field of complex numbers $C$, and let $\Lambda f=0$ be a $K$-equation on $X$ with a $K$-basis of solutions $\omega_{1}, \omega_{2}$. By definition $\omega_{1}, \omega_{2}$ are holomorphic nonvanishing multivalued functions on a Zariski open subset $X_{0} \subset X$ with $\operatorname{Im}\left(\omega_{1} / \omega_{2}\right)>0$ on $X_{0}$ and $\mathrm{SL}_{2}(\boldsymbol{Z})$ monodromy. From $\omega_{1}, \omega_{2}$ we obtain a commutative diagram:

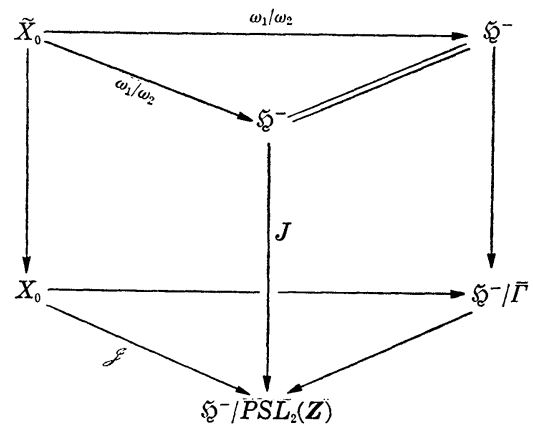

where

(i) $\tilde{X}_{0}$ is the universal cover of $X_{0}$.

(ii) $\mathfrak{S}^{-}$is the upper-half-plane minus the $\operatorname{PSL}_{2}(Z)$ orbits of a finite set of points.

(iii) $J$ is the elliptic modular function.

(iv) $\mathscr{J}=J \circ \omega_{1} / \omega_{2}$ (see $\S 0$, Proposition 0.2).

(v) $\bar{\Gamma} \subset \mathrm{PSL}_{2}(\boldsymbol{Z})$ is the projective monodromy of $\Lambda, \omega_{1}, \omega_{2}$. Note that $\bar{\Gamma}$ has finite index in $\mathrm{PSL}_{2}(\boldsymbol{Z})$ (Stiller [12]).

(vi) $\mathfrak{S}^{-} / \mathrm{PSL}_{2}(\boldsymbol{Z})$ is $\boldsymbol{P}_{\boldsymbol{C}}^{1}$ minus a finite set of points.

Two remarks are in order. First, we will take $X_{0}$ small enough to insure that $\Lambda$ will be holomorphic on $X_{0}$. Then neither $\omega_{1}$ nor $\omega_{2}$ 
vanish on $X_{0}$ and also the Wronskian $W=\omega_{1} \omega_{2}^{\prime}-\omega_{2} \omega_{1}^{\prime}=$ $\exp \left(-\int P d x\right)$ will be nonvanishing. Thus the map

$$
\widetilde{X}_{0} \stackrel{\omega_{1} / \omega_{2}}{\longrightarrow} \mathfrak{F}^{-}
$$

will be locally biholomorphic. Moreover it will be onto $\mathfrak{S}^{-}$where we have removed the $\mathrm{PSL}_{2}(\boldsymbol{Z})$ orbits of a finite set of points (Stiller [12]). In fact it will be enough to remove the points where $\mathscr{J}=0,1, \infty$, $\lambda^{2}=0, \infty$, or ord $d \mathscr{J} \neq 0$. From the explicit form of the equation (see $\S 0$, Theorem 0.3 ) one sees that choice of derivation $d / d x$ does not effect the map (1.1). Our second remark is that the triple $\Lambda, \omega_{1}, \omega_{2}$ corresponds to a unique basic elliptic surface $E$ over $X$ with functional invariant $\mathscr{J}$ and homological invariant $G$ given by the monodromy representation of $\Lambda$ for the basis $\omega_{1}, \omega_{2}$. Moreover, if $\Lambda^{\prime}, \omega_{1}^{\prime}, \omega_{2}^{\prime}$ also gives rise to $E$ over $X$ then there is a $g \in K(X)$ such that $\Lambda^{\prime}=$ $\Lambda_{g}=\Lambda_{(\mathcal{f}, g \lambda)}$ where $\Lambda=\Lambda_{(\mathcal{Z}, \lambda)}$ (see $\S 0$, Theorem 0.3) and $\omega_{i}^{\prime}=g \omega_{i}$ up to the action of $\mathrm{SL}_{2}(\boldsymbol{Z})$ changing bases. Thus the map (1.1) depends only on $E / X$.

Definition 1.1. The map $X_{0} \rightarrow \mathfrak{S C}^{-} / \bar{\Gamma}$ will be called the period map.

THEOREM 2.1. The period map $X_{0} \rightarrow \mathfrak{F}^{-} / \bar{\Gamma}$ is algebraic and extends to a regular map $X \rightarrow M_{\bar{\Gamma}}$ where $M_{\bar{\Gamma}}$ is the modular curve $\mathfrak{S}^{*} / \bar{\Gamma}$ and where $\mathfrak{S}^{*}=\mathfrak{S} \cup\{\boldsymbol{Q}\}$.

Proof. Let $x \in X-X_{0}$. Choose a disc about $x$ in $X$ with local parameter $t$ and select branches of $\omega_{1}, \omega_{2}$ single-valued in a fixed sector of the disc. Now because $A$ has regular singular points there is an integer $N$ such that $t^{N} \omega_{1} / \omega_{2}$ remains bounded as $t \rightarrow 0$ in the sector. It follows easily from this pole-like behavior that the map is algebraic and it must extend as both $X$ and $M_{\bar{\Gamma}}$ are complete smooth curves.

Note that if the corresponding elliptic surface $E / X$ has a singular fibre at $x \in X-X_{0}$ of type $I_{b}$ or $I_{b}^{*} b \geqq 1$ then the local monodromy of $\Lambda$ will be parabolic and $x$ will map to a cusp, and for types II, II*, III, III*, IV, IV* $x$ will map to an elliptic point. The only other possibility is local monodromy $\pm\left(\begin{array}{ll}1 & 0 \\ 0 & 1\end{array}\right)$ which yields some noncusp. (See Kodaira [5] for a description of these fibre types.)

From the commutative diagram above we can obtain immediate results: 
Proposition 1.3. $\left[\mathrm{PSL}_{2}(\boldsymbol{Z}): \bar{\Gamma}\right] \mid$ valence $\mathscr{J}$, that is, the index of the projective monodromy group divides the valence of $\mathscr{J}$.

Proof. Obvious.

REMARK. Using the formulas for the numerical invariants of a basic surface (Kodaira [6]), one can show, for example, that if $E / P_{c}^{1}$ is a $K_{3}$ surface then valence $\mathscr{J} \leqq 24$. It follows that the monodromy has index $\leqq 48$. This in turn limits the nature of the $K(X)$-rational torsion on $E^{\text {gen }}$ as an elliptic curve over $K(X)$.

THEOREM 1.4. Suppose $X_{0} \stackrel{\mathscr{J}}{\rightarrow} \mathfrak{S C}^{-} / \mathrm{PSL}_{2}(\boldsymbol{Z})$ (or $\left.X \stackrel{\mathscr{J}}{\rightarrow} \boldsymbol{P}_{\boldsymbol{C}}^{1}\right)$ is abelian Galois with no 2 or 3 torsion. Then $\bar{\Gamma}=\operatorname{PSL}_{2}(Z)$.

Proof. Under the hypotheses of the theorem, a standard fact in the theory of modular functions gives $\bar{\Gamma}$ normal in $\operatorname{PSL}_{2}(\boldsymbol{Z})$. We have

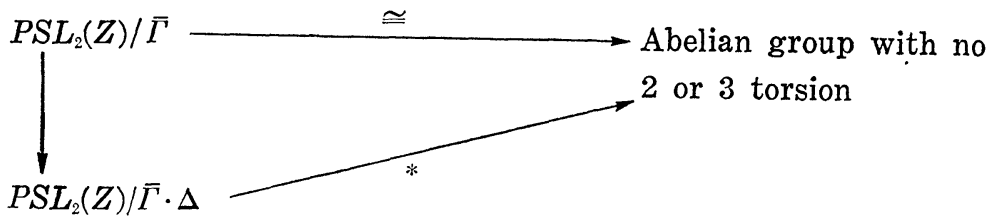

where $\Delta$ is the commutator subgroup of $\operatorname{PSL}_{2}(\boldsymbol{Z})$. But $\operatorname{PSL}_{2}(\boldsymbol{Z}) / \Delta$ surjects on $\mathrm{PSL}_{2}(\boldsymbol{Z}) / \bar{\Gamma} \cdot \Delta$ and $H_{1}\left(\mathrm{PSL}_{2}(\boldsymbol{Z}), \boldsymbol{Z}\right)=\mathrm{PSL}_{2}(\boldsymbol{Z}) / \Delta$ is $\boldsymbol{Z}_{2} \times \boldsymbol{Z}_{3}$. Thus $*$ is the zero map and $\bar{\Gamma}=\operatorname{PSL}_{2}(\boldsymbol{Z})$.

Now suppose we have a basic surface $E / X$. Assume the projective monodromy group of $E / X$ is all of $\operatorname{PSL}_{2}(Z)$.

THEOREM 1.5. Let $\hat{X} \rightarrow X$ be any abelian Galois extension of $X$ with no 2 or 3 torsion. Then the projective monodromy of $\hat{E}=$ $E \times{ }_{X} \hat{X}$ (minimal smooth model/ $\left.\hat{X}\right)$ is also $\operatorname{PSL}_{2}(\boldsymbol{Z})$.

Proof. After removing a suitable set of points from both $\hat{X}$ and $X$ we have

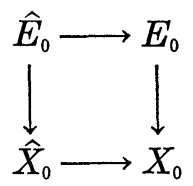

with $\hat{X}_{0} \rightarrow X_{0}$ étale Galois (thereby a covering may). Then

$$
\pi_{1}\left(\hat{X}_{0}\right) \longmapsto \pi_{1}\left(X_{0}\right) \longrightarrow \operatorname{PSL}_{2}(Z),
$$

with $\pi_{1}\left(\hat{X}_{0}\right)$ normal in $\pi_{1}\left(X_{0}\right)$ and the image of $\pi_{1}\left(\hat{X}_{0}\right)=\bar{\Gamma}$, the monodromy 
of $\hat{E}_{0}$, also normal in $\operatorname{PSL}_{2}(\boldsymbol{Z})$. So

$$
\pi_{1}\left(X_{0}\right) / \pi_{1}\left(\hat{X}_{0}\right) \longrightarrow \mathrm{PSL}_{2}(\boldsymbol{Z}) / \bar{\Gamma} \text {. }
$$

On the other hand, $\pi_{1}\left(X_{0}\right) / \pi_{1}\left(\hat{X}_{0}\right) \cong \operatorname{Gal}\left(\hat{X}_{0} / X_{0}\right)$. Thus $\operatorname{PSL}_{2}(Z) / \bar{\Gamma}$ is abelian with no 2 or 3 torsion and as above we conclude $\bar{\Gamma}=\operatorname{PSL}_{2}(\boldsymbol{Z})$.

THEOREM 1.6. Let $E / X$ be an elliptic surface over $X$ with functional invariant $\mathscr{J}$. Suppose $X \stackrel{\mathscr{J}}{\rightarrow} \boldsymbol{P}_{c}^{1}$ exhibits $X$ as a solvable Galois extension which admits a tower having no 2 or 3 torsion. Then the projective monodromy of $E / X$ is all of $\operatorname{PSL}_{2}(\boldsymbol{Z})$.

Proof. Clear.

Remark. Clearly $\bar{\Gamma}=\operatorname{PSL}_{2}(\boldsymbol{Z})$ if and only if the monodromy $\Gamma=\mathrm{SL}_{2}(\boldsymbol{Z})$. Thus $\Gamma=\mathrm{SL}_{2}(\boldsymbol{Z})$ in all the above results.

CoRollary 1.7. If the monodromy is all of $\mathrm{SL}_{2}(\boldsymbol{Z})$, as it is in the above cases, the generic fibre $E^{\mathrm{gen}} / K(X)$ as an elliptic curve over $K(X)$ has no nonzero $K(X)$-rational division points.

\section{Proof. See Stiller [12].}

Let $E / X$ be a basic surface. The homological invariant is a locally constant $Z \oplus Z$ sheaf on some $X_{0} \rightarrow X$ Zariski open. Let $x_{0} \in X_{0}$ be a base point. We can interpret the homological invariant as an action of $G=\pi_{1}\left(X_{0}, x_{0}\right)$ on $H^{1}\left(E_{x_{0}}, \boldsymbol{Z}\right)=A(\cong \boldsymbol{Z} \oplus \boldsymbol{Z})$ where $E_{x_{0}}$ is the fibre over $x_{0}$. Thus there is a map

$$
G \stackrel{\rho_{Z}}{\longrightarrow} \operatorname{Aut}_{Z}\left(H^{1}\left(E_{x_{0}}, Z\right)\right),
$$

and $A$ is naturally a $Z G$-module.

THEOREM 1.8. Let $A \otimes_{z} C \cong H^{1}\left(E_{x_{0}}, C\right)=V . \quad N o w G \stackrel{\rho_{C}}{\rightarrow} \operatorname{Aut}_{\boldsymbol{C}}(V)$, so $V$ is a $C G$-module. We claim $V$ is a simple $C G$-module, i.e., the representation is irreducible.

Proof. Say $H \subset V$ is a one dimensional invariant subspace. Thus $V$ has a basis where the representation takes the form

$$
\left[\begin{array}{ll}
* & * \\
0 & *
\end{array}\right]
$$

Now $V$ also has an underlying $\boldsymbol{Z}$-structure so that the representation 
may be taken into $\mathrm{SL}_{2}(\boldsymbol{Z})$. Hence there is an $M \in \mathrm{GL}_{2}(\boldsymbol{C})$ conjugating one form to the other. An easy computation shows that in $\mathrm{SL}_{2}(\boldsymbol{Z})$ we could get only parabolic elements fixing $i^{\infty}$ (assuming we normalize so $\pm\left[\begin{array}{ll}1 & n \\ 0 & 1\end{array}\right], n>0$ is in the monodromy group $\Gamma \subset \mathrm{SL}_{2}(\boldsymbol{Z})$. This contradicts $\Gamma \subset \mathrm{SL}_{2}(\boldsymbol{Z})$ of finite index.

We now consider when $A$ is simple as a $Z G$-module. It is easy to see that $A$ has no $G$-invariant subgroups of rank 1 . However suppose we have $H \varsubsetneqq A$ a $G$-invariant subgroup of rank 2. Obviously upon tensoring with $\boldsymbol{Q}$ we have $H \otimes_{z} \boldsymbol{Q} \cong A \otimes_{z} \boldsymbol{Q}$. If we view $A$ as $\boldsymbol{Z} \omega_{1}+\boldsymbol{Z} \omega_{2}, \omega_{1}, \omega_{2}$ a lattice for $E_{x_{0}}$, then $H$ corresponds to an invariant sublattice and clearly gives rise to another $K$-basis for our $K$-equation $A$ which is not $Z$-equivalent to $\omega_{1}, \omega_{2}$ i.e., the new basis $\omega_{1}^{\prime}, \omega_{2}^{\prime}$ is not a scalar or $\mathrm{SL}_{2}(\boldsymbol{Z})$ combination of $\omega_{1}, \omega_{2}$. This new basis for $\Lambda$ determines another elliptic surface $E^{\prime} / X$ whose generic fibre will be isogeneous to that of $E / X$. Conversely every elliptic surface $E^{\prime} / X$ which is generically isogeneous to $E / X$ over $K(X)$ arise out of another $K$-basis for 1 . (See Stiller [12] and the remarks in $\S 0$.)

2. Invariants. Let $E$ and $E^{\prime}$ be two elliptic surfaces over a common base curve $X$ with function field denoted $K(X)$. We shall assume as before that both $E$ and $E^{\prime}$ have nonconstant functional invariant, admit a section over $X$, and that they are free of exceptional curves of the first kind in the fibres. The section corresponds to a $K(X)$-rational point on the generic fibre $E^{\text {gen }}, E^{\prime \text { gen }}$. In this way $E^{\text {gen }}, E^{\prime \text { gen }}$ can be viewed as elliptic curves $/ K(X)$.

DEFINITION 2.1. $E$ and $E^{\prime}$ are said to be generically isogeneous over $X$ if the genetic fibres of $E$ and $E^{\prime}$ are isogeneous over $K(X)$.

One should note that this definition is equivalent to the existence of a rational map

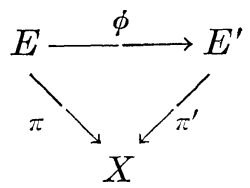

which over a Zariski open subset of $X$ is a regular fibre isogeny. Such a map need not extend to all of $E$ as examples show (see $\S 3$ ). Assume $E$ and $E^{\prime}$ are generically isogeneous and let $\phi$ be the above rational map. Choose $X_{0}$ Zariski-open in $X$ so that $E_{0}=\pi^{-1}\left(X_{0}\right)$ and $E_{0}^{\prime}=\pi^{\prime-1}\left(X_{0}\right)$ contain no degenerate fibre and so that 


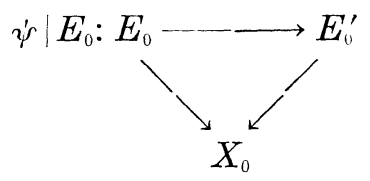

is a regular fibre by fibre isogeny. Let $D_{0}$ and $D_{0}^{\prime}$ be the GaussManin connexion (Katz and Oda [4]):

$$
D_{0}: H_{D R}^{1}\left(E_{0} / X_{0}\right) \longrightarrow \Omega_{\Lambda^{\prime} / C}^{1} \otimes_{C_{X_{0}} / C} H_{D R}^{1}\left(E_{0} / X_{0}\right)
$$

etc. for $D_{0}^{\prime}$, where $H_{D R}^{1}$ is the first hyperderived functor of direct image applied to $\Omega_{E_{0} / X_{0}}^{\cdot}$, the relative algebraic DeRham complex. Note

$$
\begin{aligned}
& H_{D R}^{1}\left(E_{0} / X_{0}\right) \cong R^{1} \pi_{*}(\boldsymbol{C}) \\
& H_{D R}^{1}\left(E_{0}^{\prime} / X_{0}\right) \cong R^{1} \pi_{*}^{\prime}(\boldsymbol{C}) .
\end{aligned}
$$

Thus we have two flat vector bundles of rank two on $X_{0}$.

THEOREM 2.2. $E$ and $E^{\prime}$; are generically isogeneous if and only if the resulting flat vector bundles are isomorphic.

Proof. See Stiller [12].

This flat bundle can be represented by a second order algebraic differential equation $X$

$$
\frac{d^{2} f}{d x^{2}}+P \frac{d f}{d x}+Q f=0
$$

where $P, Q \in K(X)$ and $x \in K(X)$ nonconstant. The resulting differential equation will be a $K$-equation $\Lambda$. However, it will possess two $K$-bases $\omega_{1}, \omega_{2}$ and $\omega_{1}^{\prime}, \omega_{2}^{\prime}$ such that $E$ will be the surface associated to $\omega_{1}, \omega_{2}$ and $E^{\prime}$ to $\omega_{1}^{\prime}, \omega_{2}^{\prime}$. Thus the functional invariant $\mathscr{J}$ of $E$ will be $J\left(\omega_{1} / \omega_{2}\right)$ and $\mathcal{J}^{\prime}$ of $E$ will be $J\left(\omega_{1}^{\prime} / \omega_{2}^{\prime}\right), J$ the elliptic modular function. Moreover the homological invariants of $E, E^{\prime}$ will correspond to the monodromy representations given by $\omega_{1}, \omega_{2}$ and $\omega_{1}^{\prime}, \omega_{2}^{\prime}$ respectively. Since both $\omega_{1}, \omega_{2}$ and $\omega_{1}^{\prime}, \omega_{2}^{\prime}$ are bases of solutions of the same differential equation the representations are complex equivalent. However they will not be equivalent over $\mathrm{SL}_{2}(\boldsymbol{Z})$. It can be shown (Stiller [12]) that there exists $M \in \mathrm{GL}_{2}{ }^{+}(\boldsymbol{Q})$ such that

$$
M\left(\omega_{1} / \omega_{2}\right)=\left(\omega_{1}^{\prime} / \omega_{2}^{\prime}\right) .
$$

Of course if $M$ is in $\mathrm{SL}_{2}(\boldsymbol{Z})$ or scalar then we will have $E \cong E^{\prime}$.

Theorem 2.3. If $E$ and $E^{\prime}$ are generally isogeneous over $X$ then the period maps commute, that is there exists a regular map $\Psi$ such that the diagram 


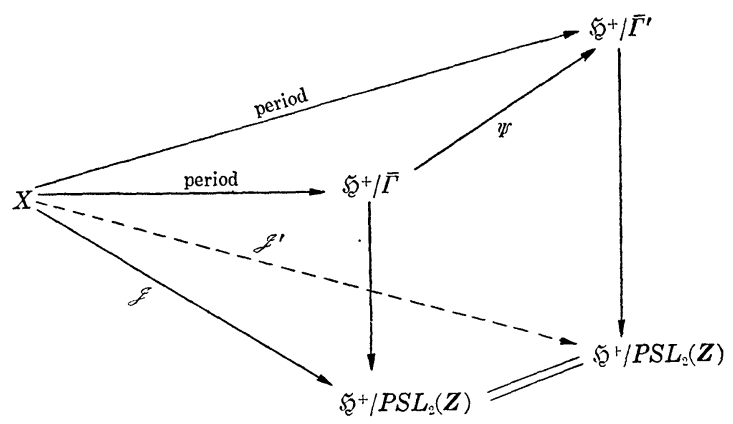

commutes. Here $\mathfrak{S}^{+}$is the upper-half-plane union the appropriate cusps and $\bar{\Gamma}, \bar{\Gamma}^{\prime}$ are the projective monodromy groups in $\operatorname{PSL}_{2}(\boldsymbol{Z})$ of $E$ and $E^{\prime}$ respectively.

Proof. Let $\rho: \pi_{1}\left(X_{0}\right) \rightarrow \mathrm{SL}_{2}(\boldsymbol{Z})$ and $\rho^{\prime}: \pi_{1}\left(X_{0}\right) \rightarrow \mathrm{SL}_{2}(\boldsymbol{Z})$ be the monodromy representations (homological invariants) of $E$ and $E^{\prime}$ respectively. For $\gamma \in \pi_{1}\left(X_{0}\right)$ we have

$$
M \rho(\gamma) M^{-1}=\rho^{\prime}(\gamma)
$$

for some $M \in \mathrm{GL}_{2}^{+}(\boldsymbol{Q})$. We have

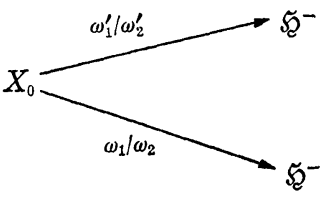

where $\mathfrak{S}^{-}$is the upper-half-plane minus a finite number of $\mathrm{SL}_{2}(\boldsymbol{Z})$ orbits, map $\mathfrak{S}^{-}$to $\mathfrak{S}^{-}$by $\tau \rightarrow M \tau$. It is easy to check that this descends to a well-defined map

$$
\mathfrak{S E}^{-} / \bar{\Gamma} \longrightarrow \mathfrak{S}^{-} / \bar{\Gamma}^{\prime}
$$

since $M \bar{\Gamma} M^{-1}=\bar{\Gamma}^{\prime}$. It then follows that the diagram commutes.

Note that $\Psi$ is an isomorphism of Riemann surfaces $M_{\bar{\Gamma}}$ and $M_{\bar{\Gamma}^{\prime}}$. Thus:

CoROLlaRY 2.4. The global projective monodromy groups must have the same index if $E$ and $E^{\prime}$ are generically isogeneous i.e., $\left[\mathrm{PSL}_{2}(\boldsymbol{Z}): \bar{\Gamma}\right]=\left[\mathrm{PSL}_{2}(\boldsymbol{Z}): \bar{\Gamma}^{\prime}\right]$.

COROLLARY 2.5. If $E$ and $E^{\prime}$ are generically isogeneous then the functional invariants $\mathcal{J}$ and $\mathcal{J}^{\prime}$ have the same valence, that is the same number of poles. 
THEOREM 2.6. If $E$ and $E^{\prime}$ are generically isogeneous then all the betti numbers $b_{i} i=0, \cdots, 4$, the geometric genus $p_{g}$ and the irregularity $q$ of $E$ and $E^{\prime}$ are the same.

Proof. We appeal to the formulas of Kodaira [6] and general relationships among the numerical invariants. Let $g$ be the genus of $X$ then $b_{1}=b_{3}=2 q=2 g$ and $b_{0}=b_{4}=1$. So only $p_{g}$ and $b_{2}$ are of interest; and quality between one of these for $E$ and $E^{\prime}$ implies equality for the other. Recall Kodaira's formula (Kodaira [6]):

$$
\begin{aligned}
12\left(p_{a}+1\right)=\mu & +6 \sum_{b \geqq 0} v\left(I_{b}^{*}\right)+2 v(\mathrm{II})+10 v\left(\mathrm{II}^{*}\right)+3 v(\mathrm{III}) \\
& +9 v\left(\mathrm{III}^{*}\right)+4 v(\mathrm{IV})+8 v\left(\mathrm{IV}^{*}\right),
\end{aligned}
$$

where $p_{a}$ is the arithmetic genus of the surface, $v(T)$ is the number of singular fibres of type $T$ (for types see Kodaira's list in Kodaira [5]), and $\mu$ is the valence of the functional invariant. As $p_{a}=$ $p_{g}-q$ it will be enough to show that the sum on the right hand side is invariant under genetic isogeny. By Corollary 2.5 above $\mu$ is invariant. Now each fibre type has an associated matrix in $\mathrm{SL}_{2}(\boldsymbol{Z})$ which represents the local monodromy up to conjugation in $\operatorname{SL}_{2}(\boldsymbol{Z})$. For example,

$$
\begin{array}{ll}
I_{b} & \left(\begin{array}{ll}
1 & b \\
0 & 1
\end{array}\right) \quad b>0 \\
I_{b}^{*} \quad\left(\begin{array}{rr}
-1 & -b \\
0 & -1
\end{array}\right) \quad b>0 \\
I_{0}^{*} \quad\left(\begin{array}{rr}
-1 & 0 \\
0 & -1
\end{array}\right) .
\end{array}
$$

Since $E$ and $E^{\prime}$ are generically isogeneous, the local monodromy at $x \in X$ of either $E$ or $E^{\prime}$ is $\mathrm{GL}_{2}(C)$-equivalent to the local monodromy for 1 . Thus trace is preserved and it follows that $\sum_{b \geq 0} v\left(I_{b}^{*}\right)$ is preserved as is $\sum_{b \geq 1} v\left(I_{b}\right)$. Note that the actual fibre type may not be preserved (see examples $\S 3$ where type $I_{2}$ becomes $I_{4}$ etc.). Of course type $I_{0}^{*}$ is preserved. In all of the remaining cases the type will be preserved. For example trace considerations show that a fibre of type II on $E$ must correspond to one of type II or II* on $E^{\prime}$. In order for the type to change there would have to be a matrix $N=$ $\left(\begin{array}{ll}a & b \\ c & d\end{array}\right)$ in $\mathrm{GL}_{2}^{+}(\mathbf{Q})$ with

$$
N\left(\begin{array}{rr}
1 & 1 \\
-1 & 0
\end{array}\right)=\left(\begin{array}{rr}
0 & -1 \\
1 & 1
\end{array}\right) N
$$

But this forces $a=-d$ and $b-a=c$. Thus $\operatorname{det} N=-a^{2}-b^{2}+a b=$ $-\left(a^{2}-a b+b^{2}\right)=-\left((a-1 / 2 b)^{2}+3 / 4 b^{2}\right) \leqq 0$ a contradiction. The 
same sort of calculation shows II*, III, III*, IV, IV* are preserved. Thus the entire sum is preserved as we desired to show.

Lastly we wish to investigate the Picard numbers $\rho$ and $\rho^{\prime}$ of $E$ and $E^{\prime}$. Recall that $\rho$ is the rank of the Néron-Severi group which is the group of divisors modulo algebraic equivalence.

THEOREM 2.7. If $E$ and $E^{\prime}$ are generically isogeneous then their Picard numbers $\rho, \rho^{\prime}$ are equal.

Proof. We make use of a formula appearing in Shioda [11]

$$
\rho=r+2+\sum_{v}\left(m_{v}-1\right) .
$$

Here $\rho$ is the Picard number, $r$ is rank of the group of $K(X)$-rational points of the generic fibre $E^{\text {gen }}, E^{\prime \text { gen }}$ which is an elliptic curve over $K(X)$, and $m_{v}$ is the number of irreducible components of the fibres where $v$ runs over the singular fibres. Since $E$ and $E^{\prime}$ are generically isogeneous $r$ is preserved. As we observed in Theorem 2.6 all fibre types are preserved except possibly $I_{b}, I_{b}^{*} b \geqq 1$. Now a fibre of type $I_{b} b \geqq 1$ has $b$ components and a fibre of type $I_{b}^{*} b \geqq 1$ has $b+4$. Let $b_{v}$ be the indices of the type $I_{b_{v}}$ that occur as $v$ runs over the singular fibres and $b_{v}^{*}$ the indices of the type $I_{b_{v}^{*}}^{*}$. Now $\sum_{v} b_{v}+\sum_{v} b_{v}^{*}$ is $\mu$ the valence of the functional invariant which is preserved. Also the number of type $I_{b}$ and the number of type $I_{b}^{*}$ are fixed by trace considerations.

We are interested in the invariance of the sum $\sum_{v} m_{v}-1$ where $v$ runs only over singular fibres of types $I_{b}, I_{b}^{*} b \geqq 1$. This becomes

$$
\sum_{v} m_{v}-1+\sum_{v^{*}} m_{v}^{*}-1
$$

where $v$ runs over types $I_{b} b \geqq 1$ and $v^{*}$ runs over types $I_{b}^{*}$. By our remarks above this is

$$
\begin{aligned}
\sum_{v} b_{v}-1+\sum_{v^{*}}\left(\left(b_{v^{*}}^{*}+4\right)-1\right) & =\sum_{v} b_{v}+\sum_{v^{*}} b_{v^{*}}^{*}-\sum_{b \geqq 1} v\left(I_{b}\right)+3 \sum_{b \geqq 1} v\left(I_{b}^{*}\right) \\
& =\mu-\sum_{b \geqq 1} v\left(I_{b}\right)+3 \sum_{b \geqq 1} v\left(I_{b}^{*}\right) .
\end{aligned}
$$

Now $\mu$ is the valence of the functional invariant and so invariant by Corollary 2.5 and the sums are invariant by trace considerations.

Thus the Picard number is invariant.

Again, we remark that under generic isogeny fibre types need not be preserved-see example in $\S 3$.

3. Computing monodromy and examples. Let $X$ be a complete smooth curve/C with function field $\boldsymbol{C}(z, w)$. Here $\boldsymbol{w}$ is given as 
an algebraic function of $z$ by an irreducible polynomial

$$
w^{n}+a_{n-1}(z) w^{n-1}+\cdots+a_{0}(z)=0, \quad a_{\imath}(z) \in \boldsymbol{C}(z) .
$$

Our purpose will be to give a method for computing the monodromy of a basic surface $E / X$ with functional invariant $\mathscr{J}=z$. We begin with the $S K$-case, i.e., $\lambda=1$.

Let $T=\left\{z_{1}=\infty, z_{2}=0, z_{3}=1, z_{4}, \cdots, z_{m}\right\}$ be the branch points of (3.1) on the $z$-sphere including $\infty, 0,1$. Pick a base point $z_{0}$ and another point $z^{*}$; letting $T^{*}=T \cup\left\{z^{*}\right\}$. We suppose given slits $L_{i}$ from $z^{*}$ to $z_{i}, i=1, \cdots, m$. Each slit has "two sides" $A_{i}, B_{i}$ oriented to run from $z^{*}$ to $z_{i}$ with $B_{i}$ being the side which maintains the sphere to the left. So $B_{1} A_{1}^{-1} B_{2} A_{2}^{-1} \cdots B_{m} A_{m}^{-1}$ is positively oriented closed curve about $z_{0}$ on the sphere.

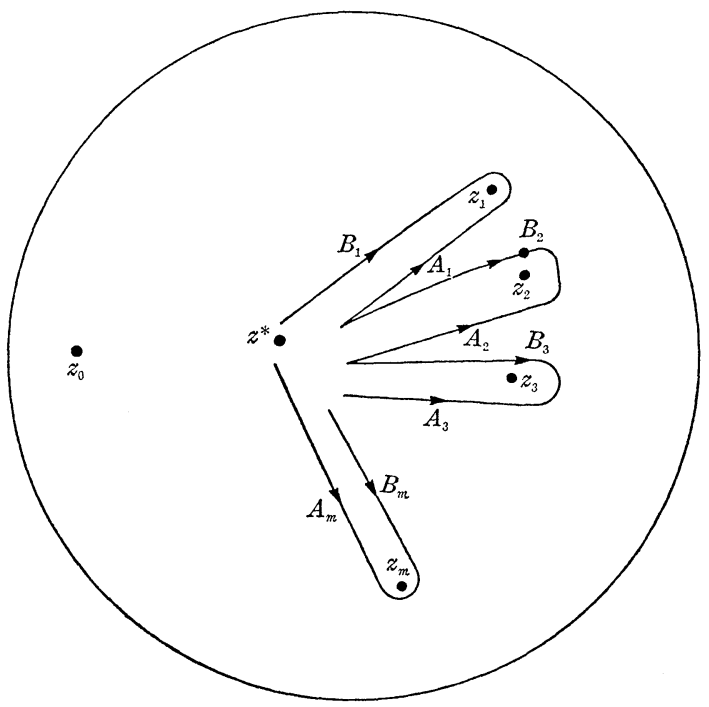

Let $w_{1}, \cdots, w_{n}$ be $n$ distinct function elements at $z_{0}$ of $w$. Assume given permutations $\pi_{1}, \cdots, \pi_{m}$ of $\{1, \cdots, n\}$ where analytic continuation of $w_{k}$ across $L_{j}$ from $B_{j}$ to $A_{j}$ leads to $w_{\pi_{j}(k)}$. Consider the free group $\Pi$ on $\pi_{1}, \cdots, \pi_{m}$, and fix a function element say $w_{1}$ at $z_{0}$. Let $X_{0}=X-$ all points over $T^{*}$.

Now we look at the $z$-sphere less $T^{*}$.

$x$

$x$

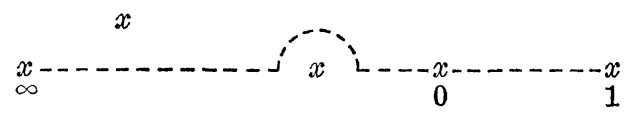


We can slit the $z$-sphere from 0 to $\infty$ and 0 to 1 so that the differential equation

$$
\frac{d^{2} f}{d z^{2}}+\frac{1}{z} \frac{d f}{d z}+\frac{((31 / 144) z-1 / 36)}{z^{2}(z-1)^{2}} f=0
$$

has single-valued solutions on the remaining part of the $z$-sphere. Further we can select a branch so that the monodromy across the slits is $\left(\begin{array}{ll}1 & 1 \\ 0 & 1\end{array}\right)$ or $\left(\begin{array}{rr}0 & 1 \\ -1 & 0\end{array}\right)$ as indicated:

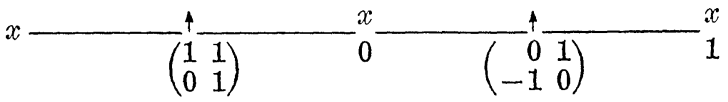

The above choices for $L_{i}, A_{i}, B_{i}, z_{0}, z^{*}$ and the slits above can all be made so that the picture becomes:

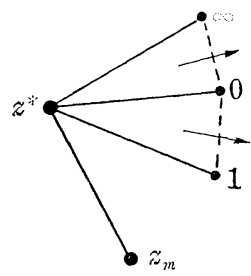

Let $\Pi_{w_{1}} \subset I I$ be all words $\gamma$ such that $1 \gamma=1$, i.e., the isotropy of 1 under the action of $\Pi$ on $\{1, \cdots, n\}$. (Note $\pi_{1} \cdots \pi_{m}$ acts like the identity.) Now let $x_{1} \cdots x_{r}$ be the points of $X$ over $T^{*}$ i.e., $X-X_{0}=$ $\left\{x_{1}, \cdots, x_{r}\right\}$. Choose words $\gamma_{1}, \cdots, \gamma_{r}$ and $C_{1}, \cdots, C_{g}, D_{1}, \cdots, D_{g}$ in the $\pi_{j}$ 's which lie in $\Pi_{w_{1}}$ and represent a basis for $\pi_{1}\left(X_{0}, w_{1} / z_{0}\right)$. Thus $\gamma_{i}$ represents a simple loop about $x_{i} ; C_{i}, D_{i}$ 's are various cycles and as permutations

$$
\prod_{i=1}^{g} C_{i} D_{i} C_{\imath}^{-1} D_{i}^{-1} \prod_{i=1}^{r} \gamma_{i}=\text { identity }
$$

THEOREM 3.1. We can select a $K=$ basis of solutions $\omega_{1}, \omega_{2}$ of our $S K$-equation on $X$ at $w_{1} / z_{0}$ so that $\mathscr{J}=J\left(\omega_{1} / \omega_{2}\right)$ and if $\gamma_{i}, C_{i}, D_{i}$ is $\pi_{f_{(1)}}^{\varepsilon_{1}} \cdots \pi_{f_{(q)}}^{s_{q}}, \varepsilon_{i}= \pm 1, f_{(i)} \in\{1, \cdots, m\}$, as a word, then the monodromy matrix for $\omega_{1}, \omega_{2}$ is:

$$
M_{f_{(1)}}^{\varepsilon_{1}} \cdots M_{f_{(q)}^{\varepsilon_{q}}}^{\varepsilon_{q}}
$$

where $M_{1}=\left(\begin{array}{ll}1 & 1 \\ 0 & 1\end{array}\right), \quad M_{2}=\left(\begin{array}{rr}1 & 1 \\ -1 & 0\end{array}\right)=\left(\begin{array}{rr}1 & -1 \\ 0 & 1\end{array}\right)\left(\begin{array}{rr}0 & 1 \\ -1 & 0\end{array}\right), \quad M_{3}=\left(\begin{array}{rr}0 & 1 \\ -1 & 0\end{array}\right)$, $M_{4}=\cdots=M_{n}=\left(\begin{array}{ll}1 & 0 \\ 0 & 1\end{array}\right)$. 
Proof. Obvious. Essentially we have pulled our single-valued branch of the solutions back to a single-valued branch on $X$ minus some slits:

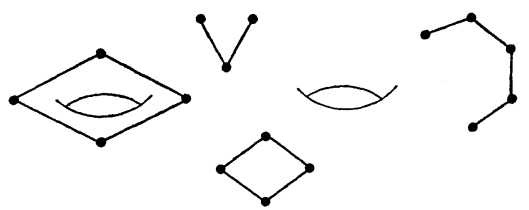

We are also able to keep track of how these solutions of our SKequation change as we cross a slit.

Now adding $\lambda$ to get the general $K$-equation amounts to changing sign around a path if $\lambda$ changes sign.

We now apply this and/or similar techniques to calculate certain global monodromy representations. Let us work on the $z$-sphere, taking $\mathscr{J}=1 /\left(1-z^{12 k}\right)$. We take the $S K$-case so that the only singular fibres are of type $I_{1}$ at the $12 k$-roots of 1 . Note $\mathscr{J}=0$ at $z=\infty$ and $\mathscr{J}=1$ at $z=0$ but the fibres are good. On $\boldsymbol{P}_{\boldsymbol{C}}^{1}-\{\infty\}$ the family is

$$
y^{2}=4 x^{3}-\frac{27}{z^{12 k}} x-\frac{27}{z^{12 k}} .
$$

Note this appears to be bad at $z=0$ but taking

$$
g=\frac{z^{3 k}}{\overline{3}} \quad \text { then } \quad g^{4} \frac{27}{z^{12 k}}=3 \quad \text { and } \quad g^{6} \frac{27}{z^{12}}=z^{6 k}
$$

So

$$
y^{2}=4 x^{3}-3 x-z^{6 k}
$$

also describes the family (Sasai [10]). As usual we take a singlevalued branch on the $\mathscr{J}$-sphere and lift:
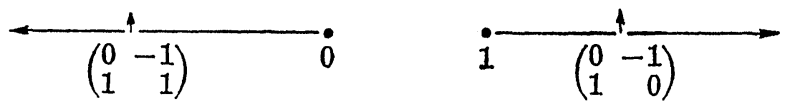

As $\mathscr{J}=1 /\left(1-z^{12 k}\right)$, the path from 0 to $\infty$ in the $\mathscr{J}$-plane lifts to the radial lines from $\infty$ to a $12 k$-root of unity, and the path from 1 to $\infty$ lifts to the radial lines from 0 to a $12 k$-root of unity. The picture is shown on the following page. 


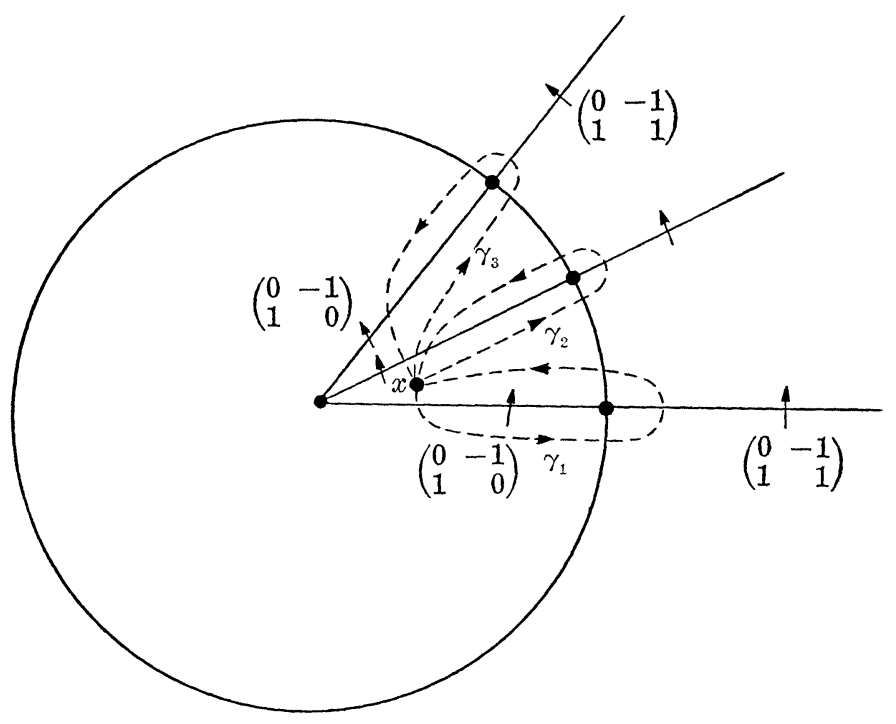

Pick a base point $x$ as marked above. Let $\gamma_{1} \cdots \gamma_{12 k}$ be the loops pictured. Then the representation is given by

$$
\begin{aligned}
\gamma_{1} \longrightarrow & \left(\begin{array}{rr}
0 & 1 \\
-1 & 0
\end{array}\right)\left(\begin{array}{rr}
0 & -1 \\
1 & 1
\end{array}\right)=\left(\begin{array}{ll}
1 & 1 \\
0 & 1
\end{array}\right) \\
\gamma_{2} \longrightarrow & \left(\begin{array}{rr}
0 & -1 \\
1 & 1
\end{array}\right)\left(\begin{array}{rr}
0 & 1 \\
-1 & 0
\end{array}\right)=\left(\begin{array}{rr}
1 & 0 \\
-1 & 1
\end{array}\right) \\
\vdots & \\
\gamma_{2 n-1} \longrightarrow & \left(\begin{array}{ll}
1 & 1 \\
0 & 1
\end{array}\right) \\
\gamma_{2 n} \longrightarrow & \left(\begin{array}{rr}
1 & 0 \\
-1 & 1
\end{array}\right) .
\end{aligned}
$$

(The reader will observe that this agrees with Sasai who obtains the result in a different manner (Sasai [10]).)

We now consider the case of $\Lambda_{(,, i)}$ where $\mathscr{J}$ is unramified over $0,1, \infty$, i.e., $X \stackrel{\mathscr{Y}}{\rightarrow} \boldsymbol{P}_{c}^{1}$ is unramified over $0,1, \infty$. This is in some sense the general case. The reader should have no trouble seeing:

Theorem 3.2. Let $X$ be any curve and $E / X$ any elliptic surface with functional invariant $\mathscr{F}$ unramified over $0,1, \infty$. Then the global monodromy group $\Gamma \subset \mathrm{SL}_{2}(\boldsymbol{Z})$ is in fact $\mathrm{SL}_{2}(\boldsymbol{Z})$.

The reader should refer to Corollary 1.7 which shows that such an $E / X$ then has no $K(X)$-rational division point on the generic fibre. 
Further, as is obvious, one can choose cycles on $X$ so that the monodromy is $\left(\begin{array}{ll}1 & 0 \\ 0 & 1\end{array}\right)$ around all cycles.

We present an example which will give us a case where the isogeny phenomenon occurs. (See Ince [3] for details on differential equations with regular singular points, local monodromy, exponents, etc.) Let

$$
\mathscr{J}=\frac{4}{27} \frac{\left(1-z+z^{2}\right)^{3}}{z^{2}(1-z)^{2}}
$$

on the $z$-sphere. $\mathscr{J}$ has a double pole at $0,1, \infty$, a triple zero at $e^{2 \pi i / 6}$ and $e^{-2 \pi i / 6}$, and a double one at $-1,2,1 / 2$. An easy calculation yields that the $S K$-equation for this $\mathscr{J}$ has exponents $\pm 1 / 2$ at $e^{2 \pi i / 6}$, $e^{-2 \pi i / 6}$ and $1 / 2,3 / 2$ at $-1,2,1 / 2$. At $0,1, \infty$ the exponents are 0,0 . Let $\lambda^{2}$ have divisor:

$$
1(\infty)+1\left(e^{2 \pi i / 6}\right)+1\left(e^{-2 \pi i / 6}\right)-1(-1)-1(2)-1(1 / 2) .
$$

The differential equation $\Lambda_{(f, \lambda)}$ with $\lambda, \mathscr{J}$ as above is holomorphic at $e^{2 \pi i / 6}, e^{-2 \pi i / 6},-1,2,1 / 2$ with exponents 0,0 at 0 and 1 and exponents $1 / 2,1 / 2$ at $\infty$. The equation is therefore the hypergeometric equation:

$$
\frac{d^{2} f}{d z^{2}}+\left(\frac{1}{z}+\frac{1}{z-1}\right) \frac{d f}{d z}+\left(\frac{1 / 4}{z(z-1)}\right) f=0
$$

which is that for ${ }_{2} F_{1}(1 / 2,1 / 2 ; 1 ; z)$. The group is easily seen to be $\Gamma(2)$ in either case (with or without $\lambda$ ). Recall $\Gamma(2)=\left\{M \in \mathrm{SL}_{2}(\boldsymbol{Z}) \mid\right.$

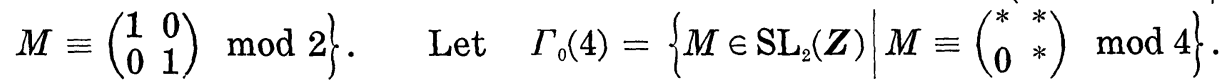
Both $\Gamma(2)$ and $\Gamma_{0}(4)$ are of index 6 in $\operatorname{SL}_{2}(Z)$ and $\left(\begin{array}{cc}1 / 2 & 0 \\ 0 & 1\end{array}\right) \Gamma(2)\left(\begin{array}{ll}2 & 0 \\ 0 & 1\end{array}\right)=$ $\Gamma_{0}(4)$. As above take

$$
\mathscr{J}=\frac{4}{27} \frac{\left(1-z+z^{2}\right)^{3}}{z^{2}(1-z)^{2}}
$$

but instead take

$$
\lambda^{2}=\frac{z-e^{2 \pi i / 6}}{z-e^{-2 \pi i / 6}} .
$$

The $K$-equation $\Lambda=\Lambda_{(f, \lambda)}$ has global group $\Gamma(2)$. The fibres are:

$\begin{array}{lllllrll}\text { at } & \infty & \text { type } & I_{2} & \text { at } & -1 & \text { type } & I_{0}^{*} \\ \text { at } & 0 & \text { type } & I_{2} & \text { at } & 2 & \text { type } & I_{0}^{*} \\ \text { at } & 1 & \text { type } & I_{2} & \text { at } & 1 / 2 & \text { type } & I_{0}^{*}\end{array}$

also the valence of $\mathscr{J}$ is 6 . Applying the well-known formulas 
(Kodaira [12]) yields $p_{a}=1, p_{g}=1, q=0$, that is, the surface $E / X$ (functional invariant $\mathscr{J}$ ) is $K 3$. We need to compute the global representation. We first compute the monodromy of Equation (1.1). We consider the usual fundamental domain for $\Gamma(2)$;

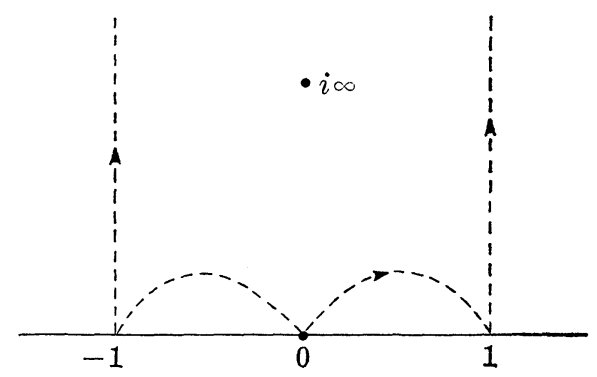

The Legendre function $\lambda$ maps this region to the $z$-sphere with $\lambda(i \infty) \rightarrow 0, \lambda(1) \rightarrow \infty, \lambda(0) \rightarrow 1$ sending the imaginary axis $i \infty$ to 0 to the slit 0 to 1 on the real axis, the arc 0 to 1 to the slit 1 to $\infty$ on the real axis, and finally the line $\operatorname{Re} \tau=1$ from 1 to $i \infty$ to the imaginary axis $\infty$ to 0 (Robert [9]). Thus if we slit the $z$-sphere along the negative real axis and from 1 to $\infty$, we will be able to find a branch of the quotient of solutions with values in this fundamental domain. Continuation across the slit is obviously
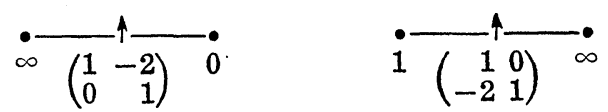

The trace is 2 (not -2 ) in both cases as the exponents at 0 and 1 are 0,0 . Choosing basis:

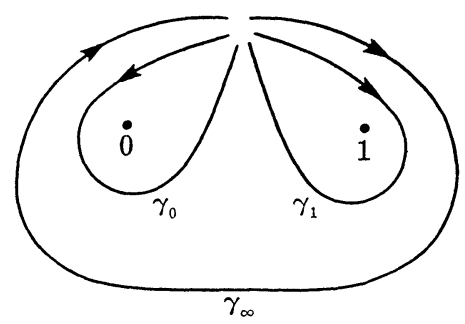

yields the representation

$$
\begin{aligned}
& \gamma_{0} \longrightarrow\left(\begin{array}{ll}
1 & 2 \\
0 & 1
\end{array}\right) \\
& \gamma_{1} \longrightarrow\left(\begin{array}{rr}
1 & 0 \\
-2 & 1
\end{array}\right) \\
& \gamma_{\infty} \longrightarrow\left(\begin{array}{ll}
1 & 0 \\
2 & 1
\end{array}\right)\left(\begin{array}{rr}
1 & -2 \\
0 & 1
\end{array}\right)=\left(\begin{array}{ll}
1 & -2 \\
2 & -3
\end{array}\right)
\end{aligned}
$$


and $\left(\begin{array}{ll}1 & 2 \\ 0 & 1\end{array}\right)\left(\begin{array}{rr}1 & 0 \\ -2 & 1\end{array}\right)\left(\begin{array}{ll}1 & -2 \\ 2 & -3\end{array}\right)=\left(\begin{array}{ll}1 & 0 \\ 0 & 1\end{array}\right)$. This is the monodromy of Equation (1.1). Note at $\infty$ the exponents are 1/2,1/2 which corresponds to trace -2 for $\left(\begin{array}{ll}1 & -2 \\ 2 & -3\end{array}\right)$. Finally putting in $\lambda$ (not to be confused with Legendre's $\lambda$ above) gives

$$
\begin{array}{r}
\gamma_{0} \longrightarrow\left(\begin{array}{ll}
1 & 2 \\
0 & 1
\end{array}\right) \\
\gamma_{1} \longrightarrow\left(\begin{array}{rr}
1 & 0 \\
-2 & 1
\end{array}\right) \\
\gamma_{\infty} \longrightarrow\left(\begin{array}{rr}
-1 & 2 \\
-2 & 3
\end{array}\right) \\
\gamma_{-1}, \gamma_{2}, \gamma_{1 / 2} \longrightarrow\left(\begin{array}{rr}
-1 & 0 \\
0 & -1
\end{array}\right)
\end{array}
$$

which is the desired representation where the basis is:

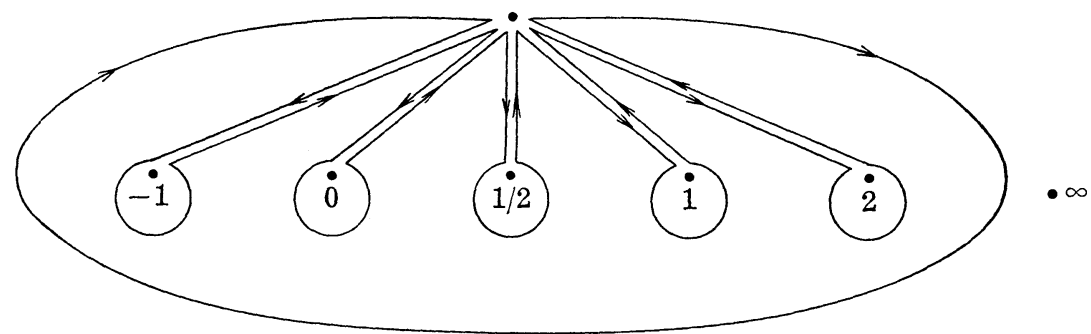

Now if $\omega_{1}, \omega_{2}$ is the $K$-basis of $\Lambda=\Lambda_{(\mathcal{F}, \lambda)}$ giving this representation $\left(\mathscr{J}=J\left(\omega_{1} / \omega_{2}\right)\right)$, then because $\left(\begin{array}{cc}1 / 2 & 0 \\ 0 & 1\end{array}\right) \Gamma(2)\left(\begin{array}{ll}2 & 0 \\ 0 & 1\end{array}\right) \subset \mathrm{SL}_{2}(Z)$ we have $\left(\begin{array}{c}\tilde{\omega}_{1} \\ \tilde{\omega}_{2}\end{array}\right)=\left(\begin{array}{cc}1 / 2 & 0 \\ 0 & 1\end{array}\right)\left(\begin{array}{l}\omega_{1} \\ \omega_{2}\end{array}\right)$ also a $K$-basis. This new basis gives another basic elliptic surface $\tilde{E} / X$ with representation:

$$
\begin{gathered}
\gamma_{0} \longrightarrow\left(\begin{array}{ll}
1 & 1 \\
0 & 1
\end{array}\right) \text { fibre type } I_{1} \\
\gamma_{1} \longrightarrow\left(\begin{array}{rr}
1 & 0 \\
-4 & 1
\end{array}\right) \text { fibre type } I_{4} \\
\gamma_{\infty} \longrightarrow\left(\begin{array}{rr}
-1 & 1 \\
-4 & 3
\end{array}\right) \text { fibre type } I_{1} \\
\gamma_{-1}, \gamma_{2}, \gamma_{1 / 2} \longrightarrow\left(\begin{array}{rr}
-1 & 0 \\
0 & -1
\end{array}\right) \text { fibre type } I_{0}^{*} .
\end{gathered}
$$

Note valence $\tilde{J}=J\left(\tilde{\omega}_{1} / \tilde{\omega}_{2}\right)$ is 6 . Thus $\widetilde{E} / X$ is also $K 3$. We have a map $E \rightarrow \widetilde{E}$ of degree 2 which is a fibre by fibre isogeny almost 
everywhere. Further the map does not extend since the number of irreducible components in the singular fibres do not agree. We remark that this also proves that $E^{\text {gen }}$ (as well as $\widetilde{E}^{\text {gen }}$ ) as an elliptic curve over $K(X)$ has a division point of order 2 rational over $K(X)$.

The reader should note that when the monodromy is all of $\operatorname{SL}_{2}(\boldsymbol{Z})$ this isogeny phenomenon does not occur and we can conclude that there are no $K(X)$-rational division points on $E^{\text {gen }}$ and the isogeny class of $E^{\text {gen }}$ over $K(X)$ contains only $E^{\text {gen }}$.

\section{REFERENCES}

1. P. Deligne, Equations Differéntielles à Points Singuliers Réguliers, Lecture Notes in Math., 163, Springer-Verlag, Berlin-Heidelberg-New York, 1970.

2. P. Griffiths, Differential Equations on Algebraic Varieties, Princeton Lectures, unpublished.

3. E. Ince, Ordinary Differential Equations, Dover Publications, New York, 1956.

4. N. Katz and T. Oda, On the differentiation of De Rham cohomology classes with respect to parameters, J. Math Kyoto Univ., 8-2 (1968), 199-213.

5. K, Kodaira, On compact analytic surfaces II, Ann. Math., 77 (1963), 563-626.

6. - On compact analytic surfaces III, Ann. Math., 78 (1963), 1-40.

7. E, Picard, Traité D'Analyse, Tome III, Gauthier-Villars, Paris, 1908.

8. H. Poincaré, Sur les Groupes des Équations Linéaires, Oeuvres, Tome II, GauthierVillars, Paris, 1916, 300-401.

9. A. Robert, Elliptic Curves, Lecture Notes in Math., 326, Springer-Verlag, Berlin-Heidelberg-New York, 1970.

10. T. Sasai, Monodromy representations of homology of certain elliptic surfaces, J. Math. Soc. Japan, Vol. 26, No. 2, (1974), 296-305.

11. T. Shioda, On elliptic modular surfaces, J. Math. Soc. Japan, Vol. 24 No. 1, (1972), 20-59.

12. P. Stiller, Differential equations associated to elliptic surfaces, to appear.

13. - Elliptic curves over function fields and the Picard number, to appear.

14. A. Weil, Généralization des fonctions abéliennes, J. Math. Pures., Appl., 17 (1938), $47-87$.

Received March 21, 1979. Supported in part by NSF Grant MCS 78-02329.

Texas A \& M University

College, TX 77843, 


\section{PACIFIC JOURNAL OF MATHEMATICS}

\section{EDITORS}

DONALD BABBITT (Managing Editor)

University of California

Los Angeles, CA 90024

HUGo RossI

University of Utah

Salt Lake City, UT 84112

C. C. MOORE and ANDREW OGG

University of California

Berkeley, CA 94720
J. DUGUNDJI

Department of Mathematics

University of Southern California

Los Angeles, CA 90007

R. FinN and J. Milgram

Stanford University

Stanford, CA 94305

\section{ASSOCIATE EDITORS}
R. ARENS
E. F. BECKENBACH
B. H. NeumanN
F. WOLF
K. Yoshida

\section{SUPPORTING INSTITUTIONS}

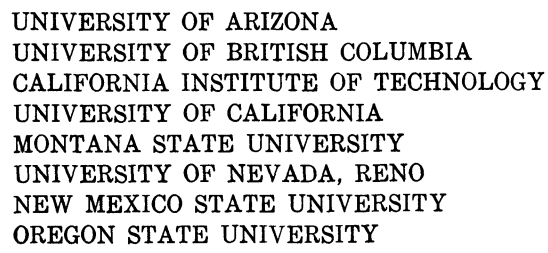

UNIVERSITY OF ARIZONA

UNIVERSITY OF BRITISH COLUMBIA CALIFORNIA INSTITUTE OF TECHNOLOGY

UNIVERSITY OF CALIFORNIA

MONTANA STATE UNIVERSITY

UNIVERSITY OF NEVADA, RENO

NEW MEXICO STATE UNIVERSITY

OREGON STATE UNIVERSITY

\author{
UNIVERSITY OF OREGON \\ UNIVERSITY OF SOUTHERN CALIFORNIA \\ STANFORD UNIVERSITY \\ UNIVERSITY OF HAWAII \\ UNIVERSITY OF TOKYO \\ UNIVERSITY OF UTAH \\ WASHINGTON STATE UNIVERSITY \\ UNIVERSITY OF WASHINGTON
}

The Supporting Institutions listed above contribute to the cost of publication of this Journal, but they are not owners or publishers and have no responsibility for its content or policies.

Mathematical papers intended for publication in the Pacific Journal of Mathematics should be in typed form or offset-reproduced, (not dittoed), double spaced with large margins. Please do not use built up fractions in the text of the manuscript. However, you may use them in the displayed equations. Underline Greek letters in red, German in green, and script in blue. The first paragraph or two must be capable of being used separately as a synopsis of the entire paper. Please propose a heading for the odd numbered pages of less than 35 characters. Manuscripts, in triplicate, may be sent to any one of the editors. Please classify according to the scheme of Math. Reviews, Index to Vol. 39. Supply name and address of author to whom proofs should be sent. All other communications should be addressed to the managing editor, or Elaine Barth, University of California, Los Angeles, California, 90024.

50 reprints to each author are provided free for each article, only if page charges have been substantially paid. Additional copies may be obtained at cost in multiples of 50 .

The Pacific Journal of Mathematics is issued monthly as of January 1966. Regular subscription rate: $\$ 102.00$ a year (6 Vols., 12 issues). Special rate: $\$ 51.00$ a year to individual members of supporting institutions.

Subscriptions, orders for numbers issued in the last three calendar years, and changes of address shoud be sent to Pacific Journal of Mathematics, P.O. Box 969, Carmel Valley, CA 93924, U.S.A Old back numbers obtainable from Kraus Periodicals Co., Route 100, Millwood, NY 10546.

\section{PUBLISHED BY PACIFIC JOURNAL OF MATHEMATICS, A NON-PROFIT CORPORATION}

Printed at Kokusai Bunken Insatsusha (International Academic Printing Co., Ltd.). 8-8, 3-chome, Takadanobaba, Shinjuku-ku, Tokyo 160, Japan. 


\section{Pacific Journal of Mathematics}

Vol. 92, No. $2 \quad$ February, 1981

Bruce Allem Anderson and Philip A. Leonard, Sequencings and Howell designs

Kevin T. Andrews, Representation of compact and weakly compact

operators on the space of Bochner integrable functions . . . . . . . . 257

James Glenn Brookshear, On the structure of hyper-real $z$-ultrafilters . . . . . 269

Frank John Forelli, Jr., A necessary condition on the extreme points of a class of holomorphic functions. II ...................... 277

Richard J. Friedlander, Basil Gordon and Peter Tannenbaum, Partitions of groups and complete mappings ......................... 283

Emden Robert Gansner, Matrix correspondences of plane partitions ......295

David Andrew Gay and William Yslas Vélez, The torsion group of a radical extension ..........................................

André (Piotrowsky) De Korvin and C. E. Roberts, Convergence theorems for some scalar valued integrals when the measure is Nemytskii ...... 329

Takaŝi Kusano and Manabu Naito, Oscillation criteria for general linear ordinary differential equations $\ldots \ldots \ldots \ldots \ldots \ldots \ldots \ldots \ldots \ldots \ldots \ldots \ldots \ldots \ldots \ldots$

Vo Thanh Liem, Homotopy dimension of some orbit spaces .......... 357

Mark Mahowald, $b o$-resolutions . . . . . . . . . . . . . . . . . . . 365

Jan van Mill and Marcel Lodewijk Johanna van de Vel, Subbases, convex

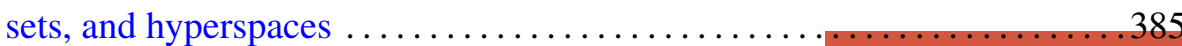

John F. Morrison, Approximations to real algebraic numbers by algebraic numbers of smaller degree $\ldots \ldots \ldots \ldots \ldots \ldots \ldots \ldots \ldots \ldots \ldots \ldots \ldots$

Caroline Series, An application of groupoid cohomology . . . . . . . . . . 415

Peter Frederick Stiller, Monodromy and invariants of elliptic surfaces . . . 433 Akihito Uchiyama, The factorization of $H^{p}$ on the space of homogeneous

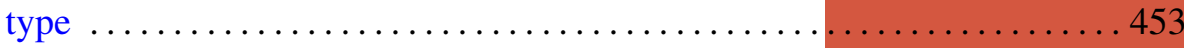

Warren James Wong, Maps on simple algebras preserving zero products.

II. Lie algebras of linear type 